\title{
Fundamentos teórico-prácticos para la toma correcta de la muestra de sangre del talón para el tamiz neonatal
}

\author{
Dra. Marcela Vela-Amieva, ${ }^{*}$ M. en C. Isabel Ibarra-González, ${ }^{* *}$ Q.F.B. Cynthia Fernández-Lainez, ${ }^{*}$ Dra. \\ Leticia Belmont-Martínez*
}

\section{RESUMEN}

El tamiz neonatal (TN) es un examen preventivo cuyo beneficio ha sido demostrado científicamente. En todos los países desarrollados el tamiz se realiza con cuatro o cinco gotas de sangre obtenidas mediante la punción del talón del recién nacido con una lanceta; se recolectan en un papel filtro especial (tarjeta de Guthrie). A pesar de su aparente sencillez, a los laboratorios de tamiz llegan muchas muestras mal tomadas o inadecuadas para realizar determinaciones bioquímicas. El objetivo del presente artículo es ofrecer recomendaciones basadas en evidencias científicas para tomar de manera correcta las muestras de sangre en papel filtro para el TN.

Palabras clave: Tamiz neonatal, sangre en papel filtro, tarjeta de Guthrie, toma de muestra, talón, lancetas.

\section{ABSTRACT}

Newborn screening (NBS) is a preventive study whose benefit has been established by solid scientific evidence. In most developed countries screening is done with 5 or 6 blood spots obtained by the puncture of the plantar surface of the infant's heel with a sterile lancet, and collected in a special filter paper (Guthrie's card). Despite its apparent simplicity, NBS laboratories commonly receive a large number of samples collected incorrectly and technically unsuitable for perfor4ming biochemical determinations. The aim of the present paper is to offer recommendations based on scientific evidence, for the properly blood collection on filter paper for NBS programs.

Key words: Newborn screening, paper filter, Guthrie cards, blood collection, heel puncture, sterile lancet.

A pesar de que el tamiz neonatal (TN) se realiza desde hace casi 50 años ${ }^{1}$ y no obstante su aparente sencillez, es frecuente que los laboratorios reciban muestras en papel filtro mal tomadas, inadecuadas para el procesamiento analítico de buena calidad. Cuando un laboratorio de tamiz recibe

\footnotetext{
* Laboratorio de Errores Innatos del Metabolismo y Tamiz del Instituto Nacional de Pediatría, SSA.

** Unidad de Genética de la Nutrición, Instituto de Investigaciones Biomédicas, UNAM.
}

Correspondencia: Dra. Marcela Vela-Amieva. Laboratorio de Errores Innatos del Metabolismo y Tamiz del Instituto Nacional de Pediatría. Av. Imán No. 1-piso 9, Col.Insurgentes-Cuicuilco, Delegación Coyoacán, CP 04530, México DF. Tel. 56-06-32-31 e-mail: dravelaamieva@yahoo.com.

Recibido: agosto, 2012. Aceptado: octubre, 2012.

Este artículo debe citarse como: Vela-Amieva M, Ibarra-González I, Fernández-Lainez C, Belmont-Martínez L. Fundamentos teóricoprácticos para la toma correcta de la muestra de sangre del talón para el tamiz neonatal. Acta Pediatr Mex 2012;33(6):273-278.

www.nietoeditore.com.mx un espécimen inadecuado, debe solicitar otro, y hay que repetir el proceso del TN. Estas muestras de mala calidad elevan el costo del TN, causan trauma innecesario a los recién nacidos $(\mathrm{RN})$, producen ansiedad en los padres, potencialmente retrasan el diagnóstico y el inicio del tratamiento de los niños afectados y lo más grave, es que pueden contribuir a pasar por alto casos con un error innato del metabolismo.

En todos los laboratorios de TN, el tiempo es un factor fundamental para que los resultados permitan iniciar oportunamente los tratamientos necesarios y prevenir las consecuencias adversas de la enfermedad.

La única justificación para rechazar una muestra y considerarla inaceptable, es que a criterio del laboratorio, no exista certeza de que su análisis bioquímico será confiable. Cuando una muestra se procesa, el laboratorio está dando por hecho que es adecuada para la prueba y asume la responsabilidad de los valores analíticos de la misma. ${ }^{2}$

Por lo anterior, es importante que el personal de salud involucrado con el TN conozca los fundamentos de la toma de muestra de sangre del talón de los RN, 
para que puedan realizarla de manera correcta y sin complicaciones.

\section{SITIOS ANATÓMICOS IDEALES PARA LA OBTENCIÓN DE MUESTRAS PARA EL TN}

En el paradigmático artículo sobre el origen del TN, Robert Guthrie textualmente expresó: "Me di cuenta que una manera simple de recolectar las gotas de sangre total en el papel filtro era puncionar el talón de los niños y depositar las gotas salientes en el papel filtro". ${ }^{3}$ Por eso desde los inicios de los programas de pesquisa, las muestras obtenidas del talón han sido preferidas por encima de cualquier otro sitio anatómico.

Los lineamientos actuales para definir el sitio ideal de punción del talón se basan en un estudio de Blumenfeld et al., en el que se recomienda que las muestras capilares se tomen de las áreas laterales de la superficie plantar del talón, para evitar lesionar al hueso calcáneo. ${ }^{4}$ Blumenfeld y su grupo estudiaron la histología de las punciones en la piel y tejidos del talón en necropsias de 40 niños; midieron la distancia entre la piel y el calcáneo y observaron que en los niños con menos de $1 \mathrm{~kg}$ de peso, dicha distancia fluctuaba entre 2.4 y $4.0 \mathrm{~mm}$ y en quienes pesaban entre 2 y $4 \mathrm{~kg}$, era de $5.0 \mathrm{~mm}$. También observaron que cuando se puncionaba dos o más veces en el mismo sitio, los tejidos se inflamaban intensamente y en algunos casos, se infectaban.

Las recomendaciones de Blumenfeld para realizar la punción del talón son: 1) La punción debe hacerse en la porción más lateral de la superficie plantar del talón. 2) No debe exceder de $2.4 \mathrm{~mm}$ de profundidad. 3) No debe hacerse en la curvatura posterior del talón. 4) No debe hacerse en sitios previamente puncionados, pues se consideran potencialmente infectados. (Figura 1).

Después del estudio clásico de Blumenfeld y colaboradores, otros autores han descrito la distancia que hay entre la piel y el calcáneo, y han llegado a conclusiones similares. Jain y Rutter analizaron ultrasonográficamente el talón de 80 RN, concluyeron que el trayecto más corto entre la piel y el calcáneo se encuentra en el centro del talón y que existe una discreta correlación entre la distancia y el peso del niño; que dicha distancia iba de 3.0 a $8.0 \mathrm{~mm}$, y reafirmaron que las punciones no deben ser mayores a $2.4 \mathrm{~mm}$ de profundidad, para evitar lastimar el calcáneo.

${ }^{5}$ El que los bordes laterales del talón son los sitios ideales

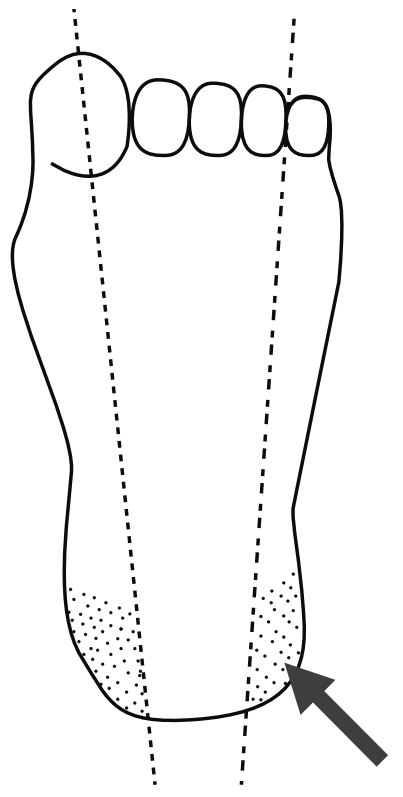

Figura 1. Esquema de Blumenfeld. Las líneas punteadas son imaginarias; una va desde la mitad del primer dedo hacia el talón y la otra, desde el pliegue interdigital del cuarto y quinto dedos hacia el talón. Las zonas marcadas con puntos en los bordes laterales del talón, señalan las áreas adecuadas para realizar la punción. La flecha indica un sitio ideal para hacerla.

para realizar la punción ha sido corroborada por varios investigadores. ${ }^{6,7}$

Las puntas de los dedos nunca deben ser utilizadas para obtener las muestras de sangre, puesto que la distancia de entre la piel y los huesos puede ser tan corta como $1.2 \mathrm{~mm}$ y se pueden lesionar las falanges. Se han descrito casos de gangrena después de una punción. ${ }^{4}$ Otros sitios, como los lóbulos de la oreja no deben utilizarse; pueden causar sangrado excesivo. ${ }^{2}$

\section{INSTRUMENTOS PARA LA PUNCIÓN DEL TALÓN}

Lo ideal, es utilizar lancetas automáticas especialmente diseñadas para el TN, que a diferencia de las agujas cónicas, hacen pequeñas incisiones de $1.0 \mathrm{~mm}$ de profundidad por 2.5 de largo. Existen muchos modelos; los hay para los RN prematuros o de bajo peso. Independientemente de la lanceta que se utilice, la punción nunca debe exceder $2.00 \mathrm{~mm}$ de profundidad. ${ }^{2}$

Las agujas y otros instrumentos cortantes, por ejemplo hojas de bisturí, deben evitarse, puesto que no tienen "freno" alguno y penetran distancias más allá de lo re- 
comendado; elevan el riesgo de complicaciones graves (Figuras 2 A y B).
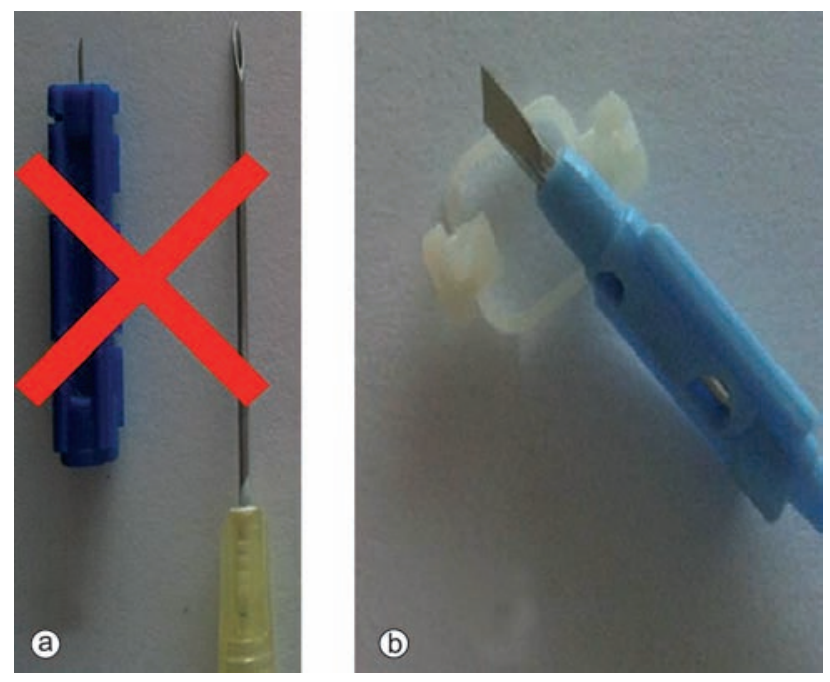

Figura 2. A. Las agujas no deben utilizarse pues al no tener freno, penetran más de $2.4 \mathrm{~mm}$. Las lancetas delgadas de punta redonda, no producen buen flujo sanguíneo. B. Lanceta automática desarmada, que muestra una pequeña cuchilla para producir cortes adecuados para el TN.

\section{PRINCIPALES COMPLICACIONES DE LA PUNCIÓN DEL TALÓN}

La complicación más frecuente, por mala técnica de punción, es el sangrado insuficiente que impida recolectar todas las gotas requeridas. Esto obliga a repetir el proceso.

Se han descrito complicaciones mucho más graves: Lilien y colaboradores publicaron un caso de osteomielitis del calcáneo como consecuencia de la punción del talón ${ }^{8}$. Otros investigadores han documentado casos similares. ${ }^{9-13}$ Estas complicaciones se pueden evitar usando una buena técnica de toma de muestra, con las medidas de higiene convencionales y con lancetas estériles adecuadas, puncionando siempre en los bordes laterales del talón y evitando puncionar dos o más veces en el mismo sitio.

Una eventualidad mucho más rara es el desarrollo de nódulos calcificados en el sitio de la punción; suelen aparecer varios meses o años después de realizada la herida y son más frecuentes cuando el talón se ha sometido a punciones múltiples. El tratamiento de esta complicación suele ser la escisión quirúrgica del nódulo. ${ }^{14-16}$
En algunos programas de TN se recomienda calentar el pie del RN para favorecer el flujo capilar y obtener mejores gotas de sangre del talón; sin embargo, esta práctica tiene riesgos pues se han descrito quemaduras del pie por someterlo a calor para tomar la muestra ${ }^{17}$. Una publicación reciente menciona el caso de un niño, cuyo pie fue quemado con una secadora de pelo a fin de "calentar el pie para tomar el tamiz". ${ }^{18}$ Debido a estos riesgos, nosotros no recomendamos calentar el pie; basta con dar un masaje suave al talón y utilizar las técnicas adecuadas de toma de muestra. No se deben utilizar toallas calientes, lámparas ni otros artefactos. El calentamiento previo es innecesario en los prematuros y en los niños de término, pues además de peligroso, aumenta el costo e incrementa el tiempo empleado en la de toma del tamiz. ${ }^{19}$

\section{OBTENCIÓN DE LA SANGRE MEDIANTE VENOPUNCIÓN}

En algunas situaciones específicas se puede tomar la muestra de sangre para el TN de una vena periférica; la más recomendada es la vena dorsal de la mano. ${ }^{2}$

Si el RN está enfermo y se le ha canalizado una vena para recibir soluciones o medicamentos, no deben utilizarse las venas de la misma mano para obtener la muestra para el tamiz neonatal, pues las soluciones pueden diluir la muestra o interferir con el análisis bioquímico.

Las muestras venosas para el tamiz, deben obtenerse mediante catéteres infantiles (mariposas), que pueden acortarse para recolectar fácilmente las gotas en el papel tamiz. No se deben utilizar jeringas puesto que el paso de la aguja a la jeringa seguido por su colocación en el papel filtro propician la formación de coágulos y la hemólisis de la sangre, lo que da muestras no válidas para el procesamiento analítico. ${ }^{2}$

Bioquímicamente, la sangre venosa es adecuada para hacer el TN, pero la mayoría de los programas utilizan la sangre del talón. En una interesante Revisión Cochrane ${ }^{20}$ cuyo objetivo fue determinar si la venopunción era menos dolorosa y más efectiva para la toma de muestras de sangre en el RN de término; se concluyó que la venopunción, realizada por un flebotomista experto, parece ser mejor que la punción del talón, pues brinda mejores muestras de sangre y es menos dolorosa; sin embargo, se requieren más estudios controlados aleatorizados para una recomendación definitiva. 


\section{RECOMENDACIONES PARA ATENUAR EL DOLOR DE LA PUNCIÓN}

Algunos autores recomiendan la administración oral de soluciones azucaradas antes de realizar la punción con lanceta, para mitigar el dolor. ${ }^{21,22}$

Una Revisión Cochrane sobre este tema, concluye que la sacarosa (azúcar común) es segura y reduce el dolor; sin embargo, no se ha determinado la dosis adecuada puesto que existen diferentes protocolos. Las dosis utilizadas van de 0.012 a 0.12 g; por otro lado, no se han analizado las consecuencias de esta práctica en recién nacidos de bajo peso, enfermos o con ventilación mecánica, por lo que hay que utilizarlas con cuidado. ${ }^{23}$ Con fines de analgesia, en los RN de término, estas soluciones de sacarosa se preparan al 24\% (24 gramos de azúcar en $100 \mathrm{~mL}$ de agua) y se proporcionan de 0.5 a $2 \mathrm{~mL}$ unos minutos antes de la punción, por vía oral de preferencia con un chupón. ${ }^{24}$

\section{ABC DE LA TOMA DE SANGRE}

1. Se deben tener todos los insumos a la mano: papel filtro (tarjeta de Guthrie) con todos los datos demográficos en la ficha de identificación, las lancetas, las torundas con alcohol, guantes y algodón seco o bandita adhesiva para detener el sangrado.

Durante casi cinco décadas, el papel filtro que se ha utilizado para recolectar las muestras, seleccionado en casi todos los países como el medio ideal para el depósito de la sangre, es el tipo Whatman 903 (antes denominado Schliecher \& Schuell 903), similar al utilizado por Guthrie en 1963. La ventaja de este papel es que está hecho con $100 \%$ de algodón, es un medio de transporte inerte, permite la recolección uniforme y conserva la sangre y la elución posterior de los analitos. Actualmente la tarjeta de Guthrie suele unirse a la ficha de identificación con los datos relevantes del binomio madre/hijo; la información de identificación y la muestra siempre están juntas.

2. Identificar el área a puncionar recordando las recomendaciones de Blumenfeld (Figura 1).

3. Inmovilizar gentilmente el pie, apoyando los dedos en el tobillo del RN.

4. Limpiar el área a puncionar con algodón impregnado de alcohol y dejar evaporar el exceso. No se debe utilizar antiséptico yodado.
5. Utilizar de preferencia una lanceta automática. En el caso de lancetas manuales, introducir la punta de la lanceta en el talón con un sólo movimiento rápido y seguro en dirección casi perpendicular a la superficie del pie. Si se usa la lanceta automática, se coloca y se activa el mecanismo. Hay que tener cuidado de no exprimir el área vecina ya que se produciría hemólisis y se puede mezclar el líquido intersticial con las gotas de sangre. Si la sangre no fluye, se coloca el pie por debajo del nivel del corazón y se frota la pierna para producir una mayor afluencia de sangre al talón.

6. Poner en contacto la superficie de la tarjeta de papel filtro con la gota de sangre y dejar que se impregne por completo el círculo. Cuidar que la piel no toque la tarjeta. La gota debe ser suficientemente grande para saturar el círculo completo e impregnar hasta la cara posterior de la tarjeta de papel filtro. Esperar una nueva gota y poner nuevamente la tarjeta en contacto con la gota de sangre para llenar el segundo círculo. Repetir el mismo procedimiento hasta completar perfectamente todos los círculos de la tarjeta de Guthrie (Figura 3).

7. Una vez completada la toma de gotas de sangre, levantar el pie del niño por arriba del nivel del corazón y presionar el área de la punción con un algodón limpio o colocar una bandita adhesiva.

8. Dejar secar la tarjeta de papel filtro y procurar no tocar con los dedos los círculos que contienen las gotas de sangre. Habitualmente las muestras se secan en dos o tres horas, dependiendo de la temperatura y humedad del ambiente.

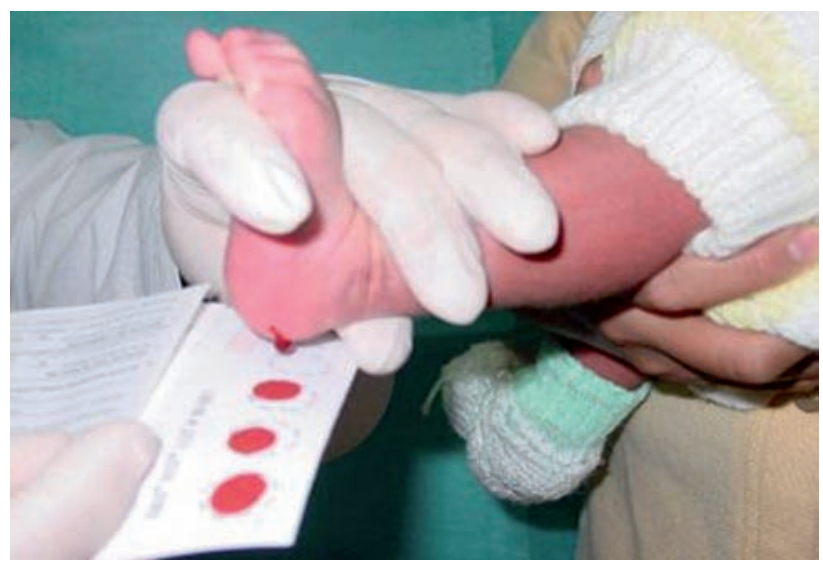

Figura 3. Posición sugerida de las manos del operador, para obtener un buen flujo sanguíneo. 
9. Una vez seca, se introduce en el sobre correspondiente para su envío inmediato al laboratorio de tamiz. Si se utiliza sobre de plástico, colocar un desecante en cada bolsa.

\section{EVALUACIÓN DE LAS MUESTRAS}

En una muestra bien tomada, las gotas de sangre son de suficiente tamaño para saturar el círculo completo e impregnar la cara posterior de la tarjeta de papel filtro.

La Figura 4 muestra algunos ejemplos de muestras mal tomadas. Es importante recordar que el mejor evaluador de la muestra es el propio operador, y es preferible repetir la punción en el mismo momento, que esperar a que la muestra sea rechazada y se tenga que volver a llamar al niño.
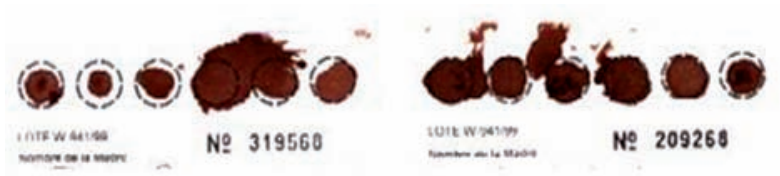

Sobresaturada con coágulos
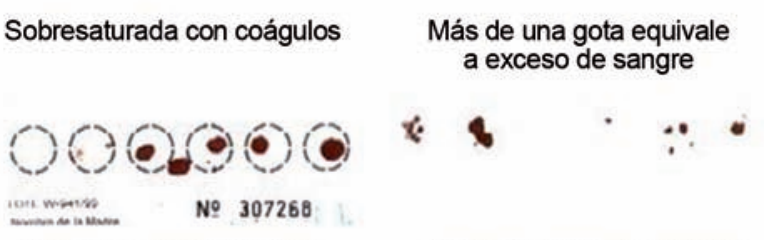

Escasa, la sangre no lleno el círculo

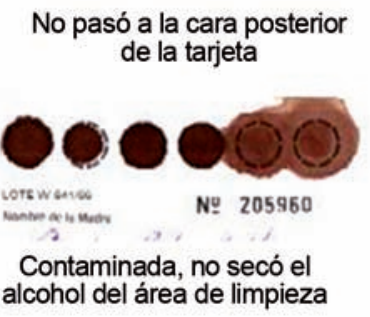

Figura 4. Ejemplos de muestras mal tomadas.

\section{CONCLUSIÓN}

Las muestras de sangre para el TN deben obtenerse mediante la punción de los bordes laterales del talón de los RN. Para ello hay que utilizar lancetas especiales para RN cuya penetración en la piel no sea mayor de $2.4 \mathrm{~mm}$ en los niños de término y $2.0 \mathrm{~mm}$ en los prematuros. Para evitar complicaciones, no deben utilizarse agujas ni otros instrumentos punzocortantes. No es recomendable calentar el pie, ni debe aplicarse la lanceta en un sitio previamente puncionado.

\section{REFERENCIAS BIBLIOGRAFICAS}

1. Guthrie R, Susi A. A simple phenylalanine method for detecting phenylketonuria in large populations of newborn infants. Pediatrics 1963;32:338-43.

2. Hannon HW, Whitley RJ, Davin B, fernhoff P, Halonen T, Lavochkin $\mathrm{M}$, et al. Clinical and Laboratory Standards Institute (CLSI)Blood Collection On Filter Paper For Newborn Screening Programs. Approved Standard -Fifth Edition. CLSI document LA4-A5 (ISBN 1-56238-644-1). Clinical and Laboratory Standards Institute. Pennsylvania: 2007.

3. Guthrie R. The origin of newborn screening. Screening 1992;1:5-15.

4. Blumenfeld TA, Turi GK, Blanc WA. Recommended site and depth of newborn heel skin punctures based on anatomical measurements and histopathology. Lancet 1979;3:230-3.

5. Jain A, Rutter N. Ultrasound study of heel to calcaneous depth in neonates. Arch Dis Child Fetal Neonatol Ed 1999;80:F243-5.

6. Reiner CB, Meites S, Hayes JR. Optimal sites and depths for skin puncture for infants as assessed from anatomical measurements. Clin Chem 1990;36:547-9.

7. Arena J, Emparanza JI, Nogués A, Burls A. Skin to calcaneus distance in the neonate. Arch Dis Child Fetal Neonatal Ed 2005;90(4):F328-31.

8. Lilien LD, Harris VJ, Ramamurthy RS, Pildes RS. Neonatal osteomyelitis of the calcaneus: complication of heel puncture. J Pediatr 1976;88(3):478-80.

9. Uhren R, Curtis P. Calcaneal osteomyelitis of the newborn: a case report. J Fam Pract 1980;11(5):809-10.

10. Canale ST, Manugian AH. Neonatal osteomyelitis of the os calcis: a complication of repeated heel punctures. Clin Orthop Relat Res 1981;156:178-82.

11. Goldberg I, Shauer L, Klier I, Seelenfreund M. Neonatal osteomyelitis of the calcaneus following a heel pad puncture: a case report. Clin Orthop Relat Res 1981;(158):195-7.

12. Gjurić G, Nikolic E, Benjak V, Mardesić D, Sarnavka V. Osteomyelitis as a complication of capillary blood collection for neonatal screening. Screening 1992;1:243-7.

13. Yüksel $S$, Yüksel G, Oncel $S$, Divanli E. Osteomyelitis of the calcaneus in the newborn: an ongoing complication of Guthrie test. Eur J Pediatr 2007;166(5):503-4.

14. Leung AK. Cutaneous nodule following heel pricks. Can Med Assoc J 1985;132(10):1163.

15. Rho NK, Youn SJ, Park HS, Kim WS, Lee ES. Calcified nodule on the heel of a child following a single heel stick in the neonatal period. Clin Exp Dermatol 2003;28(5):502-3.

16. Kurzydlo AM, Hannaford R. Dystrophic calcification following neonatal heel-prick testing. Australas J Dermatol 2010;51(3):206-7.

17. Hassan Z, Shah M. Scald injury from the Guthrie test: should the heel be warmed? Arch Dis Child Fetal Neonatal Ed 2005;90(6):F533-4.

18. Ray R, Godwin Y, Shepherd A. Convective burn from use of hairdryer for heel warming prior to the heel prick test - a case report. BMC Pediatr 2010;11:30.

19. Janes M, Pinelli J, Landry S, Downey S, Paes B. Comparison of capillary blood sampling using an automated incision device with and without warming the heel. J Perinatol 2002;22(2):154-8. 
20. Shah VS, Ohlsson A. Venepuncture versus heel lance for blood sampling in term neonates. Cochrane Database Syst Rev 2011;(10):CD001452.

21. Leef KH. Evidence-based review of oral sucrose administration to decrease the pain response in newborn infants. Neonatal Netw 2006;25(4):275-84.
22. Holsti L, Grunau RE. Considerations for using sucrose to reduce procedural pain in preterm infants. Pediatrics 2010;125(5):1042-7.

23. Stevens B, Yamada J, Ohlsson A. Sucrose for analgesia in newborn infants undergoing painful procedures. Cochrane Database Syst Rev 2010;(1):CD001069.

24. Joung $\mathrm{KH}, \mathrm{Cho} \mathrm{SC}$. The effect of sucrose on infants during a painful procedure. Korean J Pediatr 2010;53:790-4.

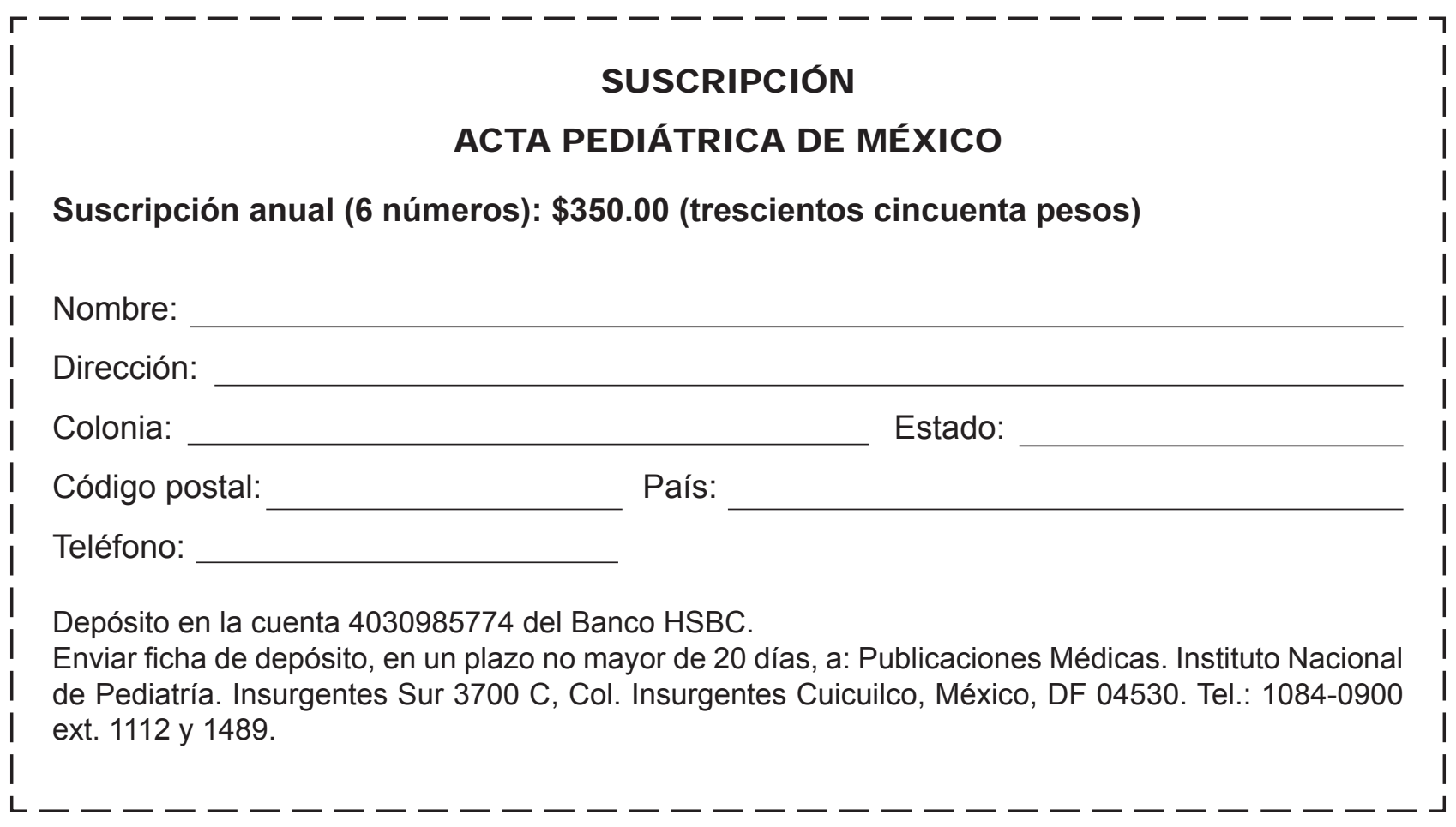

